\title{
A new sustainable symbiotic association of lactic acid cocci and bacilli for colonization/recolonization of vagina and prevention of bacterial vaginosis
}

\author{
Hrachya Garegin Hovhannisyan, Gohar Gagik Grigoryan \\ “Armbiotechnology” S\&P Center of National Academy of Sciences of Armenia, Yerevan, Armenia \\ Email address: \\ hhov@sci.am (H. G. Hovhannisyan), gohar22222@gmail.com (G. G. Grigoryan)
}

\section{To cite this article:}

Hrachya Garegin Hovhannisyan, Gohar Gagik Grigoryan. A New Sustainable Symbiotic Association of Lactic Acid Cocci and Bacilli for Colonization/Recolonization of Vagina and Prevention of Bacterial Vaginosis. Amenrican Journal of Bioscience. Vol. 2, No. 3, 2014, pp. 84-88. doi: 10.11648/j.ajbio.20140203.11

\begin{abstract}
Various factors; nutrition, hygiene, stress, infections, low immunity, menses, frequent sexual intercourses, pregnancy etc., destroyed vaginal microbial balance, which reflected in the number of lactobacilli decrease and increase the number of pathogenic microorganisms, as well as the $\mathrm{pH}$-value. All this in turn causes vaginosis. The aim of this study is creation of symbiotic association of probiotic bacteria (SAPB) with high colonization/recolonization properties for rapid response to vaginal acute and chronic challenges. It is expected that LABs symbiotic association will be more beneficial for vaginal colonization/recoclonization, because the lactococci are growth well at elevate $\mathrm{pH} 9.0-5.0$ and by reducing of vaginal $\mathrm{pH}$ promote the growth of lactobacilli, adapted to low $\mathrm{pH}$ 6.5-3.5. In this study used lactobacilli biocompatible with cocci possessing high antimicrobial activity from laboratory LABs collection, isolated earlier from 20-45 years old healthy Armenian woman volunteers $(n=40)$. And it is the first time that lactococci were used as probiotic in vaginal colonization/recolonization practice. The symbiotic association of Lactobacillus plantarum (L. plantarum) GH 202 and Lactococcus lactis (L. lactis) GH 204 strains shows higher inhibitory activities against bacterial pathogens and Candida albicans $(C$. albicans). The SAPB growth rate is more intensive and biomass accumulation is higher, than the mono cultures. It is sustainable at different $\mathrm{pH}$ conditions of growth and during multiple subculturing imitating up and down changes of vaginal $\mathrm{pH}$. The SAPB strains have high hydrophobicity evaluated by MATS test and high coaggregation properties essential for increasing their colonization potential, when they used in mixed culture.
\end{abstract}

Keywords: LABs Association, Antimicrobial Activity, Adhesion, Coaggregation

\section{Introduction}

Lactic acid bacteria (LAB) are considered the dominant microflora of healthy woman's vagina, they produce lactic acid, hydrogen peroxide $\left(\mathrm{H}_{2} \mathrm{O}_{2}\right)$, bacteriocins and other antibacterial substances that are inhibit pathogenic microorganisms and keep vagina $\mathrm{pH}<4,5[2,4,17]$. When lactobacilli are absent or amount dramatically reduced, resident anaerobe microorganisms begin to multiplate rapidly and cause bacterial vaginosis [11]. Various factors; nutrition, hygiene, stress, infections, low immunity, etc., destroyed vaginal microbial balance, which reflected in the number of lactobacilli decrease and increase the number of pathogenic microorganisms, as well as the $\mathrm{pH}$-value.

Moreover, there are few unifying theories to explain the ecological dynamics of vaginal ecosystems as they respond to disturbances caused by menses and human activities such as intercourse, douching, and other habits and practices. Exposure to an altered milieu will cause a fluctuation in the local environment and heighten or diminish the selective advantage of specific vaginal microbes. For example, the loss of lactobacilli from the vagina has been associated with sexual intercourse or with the use of antibiotics for non-vaginal illnesses. Over the course of the menstrual cycle, vaginal levels of hormones and glycogen vary, and menstrual blood $(\mathrm{pH}$ 7.32) alters vaginal $\mathrm{pH}$ and provides a substrate for many microorganisms. Nevertheless, levels of vaginal lactobacilli appear to remain constant throughout the cycle; non-Lactobacillus species increase during the proliferative phase, while Candida albicans concentrations are highest towards menstruation (as determined by culture) [15].

The buffer capacity of semen $(40 \mathrm{mM} / \mathrm{pH})$ dominates the 
buffer capacity of the vagina after intercourse [3]. The rate of acid production in the vagina has not been directly observed, but Masters and Johnson [19] demonstrated that the alkaline buffering action of the ejaculate ( $\square \mathrm{pH} 7.6$ ) abolishes vaginal acidity for several hours after intercourse and that the reacidification rate of the vagina after intercourse is $0.5 \mathrm{pH}$ units/h.

Lactobacilli colonization efficiency depends on their adaptive (propagation rate, epithelial cells adhesion, stability towards stress) and probiotic (intracellular interaction, antibacterial compounds synthesis; lactic acid, hydrogen peroxide, bacteriocins) properties $[6,9]$, as well as immunomodulation abilities. Lactobacillus species that produce $\mathrm{H} 2 \mathrm{O} 2$, were more likely to persist over time in the vagina than $\mathrm{H} 2 \mathrm{O} 2$-negative strains.

The efficiency of probiotics consisting of lactobacilli belonging to the same or different species is not high, because they partially or fully disappear after a few menses and/or frequent sexual intercourses.

It is expected that LABs symbiotic association will be more beneficial for vaginal colonization/recoclonization, because the lactococci are growth well at elevate $\mathrm{pH}$ 9.0-5.0 and by reducing of vaginal $\mathrm{pH}$ promote the growth of lactobacilli, adapted to low $\mathrm{pH}$ 6.5-3.5.

The aim of this study is to establish a sustainable symbiotic association of vaginal lactobacilli and lactococci possessing higher probiotic and adaptive properties.

\section{Material and Methods}

\subsection{Culture Media}

M16 (Merck, BRG), LAPTg (yeast extract, peptone, tryptone, glucose, Tween 80) broth, Nutrient triptose agar (Ferak, Berlin), Saburo agar (Himedia, India).

Solvents: Chlorophorm, xylene (Merck, BRG)

\subsection{Microorganisms}

In this study used LABs from laboratory collection isolated earlier from 20-45 years old healthy Armenian woman volunteers and identified by RAPT-PCR and API 50 CHL test (BioMerieux, Marcy l'Etoile, France).

The test microorganisms: Staphylococcus aureus MDC 5233, Escherichia coli MDC 5003, Candida albicans MDC 8013 from the Microbial Depositary Center of "Armbiotechnology" SPC, NAS of Armenia. All microorganisms were stored in milk/yeast extract (130 g non-fat milk, $5 \mathrm{~g}$ yeast extract and $10 \mathrm{~g}$ glucose 11$)$ at $-20{ }^{\circ} \mathrm{C}$.

\subsection{Culture Growth}

Bacteria were grown overnight under anaerobic conditions in test tubes, aliquots were inoculated in Erlenmeyer flasks containing $20 \mathrm{ml}$ MRS broth and grows at $37^{\circ} \mathrm{C}$ in aerobic condition with agitation up to late-stationary phase. The OD of cultures was determined at $\lambda=600 \mathrm{~nm}$ using a spectrophotometer.

\subsection{Screening for Production of Antagonistic Substances}

The effects of supernatant fluid of 40 strains of vaginal lactobacilli on the growth of pathogens were studied by employing the plate-diffusion technique [13]. Briefly, Triptose agar plates (standardized volume, $15 \mathrm{ml}$ ) with $10^{6}-10^{7}$ CFU of each pathogen were prepared. Standardized aliquots $(25 \mu 1)$ of neutralized supernatant of lactobacilli were placed with discs (standardized diameter, $5 \mathrm{~mm}$ ) in the pathogen-inoculated plates. The plates were incubated for $5 \mathrm{~h}$ at room temperature and then for $24 \mathrm{~h}$ at $37^{\circ} \mathrm{C}$. A clear inhibition zone of $>7 \mathrm{~mm}$ diameter was defined as a positive result.

\subsection{Test on Biocompatibility}

$10 \mu \mathrm{l}$ of overnight cultures for testing were dropped on LAPTg agar surface giving $3 \mathrm{~mm}$ diameter spot, dried then the second culture spotted at a distance of 1-2 mm for partially overlap of the first spot and plates incubated at $37^{\circ} \mathrm{C}$ for 24 hours. The cultures considered biocompatible if full merger of spot borders was observed.

\subsection{Microbial Adhesion to Solvents}

Microbial adhesion to solvents (MATS) was measured according to the method of Rosenberg et al. (1980) with some modifications [7, 18]. Bacteria were harvested in the stationary phase by centrifugation at $5000 \mathrm{~g}$ for $15 \mathrm{~min}$, washed twice, and resuspended in $0.1 \mathrm{M} \mathrm{KNO}_{3}(\mathrm{pH}$ 6.2) to approximately $10^{8} \mathrm{CFU} / \mathrm{ml}$. The absorbance of the cell suspension was measured at $600 \mathrm{~nm}\left(\mathrm{~A}_{0}\right)$. One milliliter of solvent was added to $3 \mathrm{ml}$ of cell suspension. After a 10-min preincubation at room temperature, the two phase system was mixed by vortexing for 2-min. The aqueous phase was removed after $20 \mathrm{~min}$ of incubation at room temperature, and its absorbance $\left(\mathrm{A}_{1}\right)$ at $600 \mathrm{~nm}$ was measured. The percentage of bacterial adhesion to solvent was calculated as $\left(1-\mathrm{A}_{1} / \mathrm{A}_{0}\right) \times 100$.

\subsection{Coaggregation Assay}

Bacteria were grown for $18 \mathrm{~h}$ at $37^{\circ} \mathrm{C}$ in LAPTg broth. The cells were harvested by centrifugation at $5000 \mathrm{~g}$ for 15 min, washed twice and resuspended in their culture supernatant fluid or in phosphate buffered saline (PBS) to give viable counts of approximately $10^{8} \mathrm{CFU} / \mathrm{ml}$. Equal volumes $(2 \mathrm{ml})$ of each cell suspension were mixed together in pairs by vortexing for $10 \mathrm{~s}$. Control tubes were set up at the same time, containing $4 \mathrm{ml}$ of each bacterial suspension on its own. The absorbance (A) at $600 \mathrm{~nm}$ of the suspensions was measured after mixing and after $5 \mathrm{~h}$ of incubation at room temperature. The percentage of coaggregation was calculated using the equation of Handley et al. [12]:

$$
\text { Coaggregation }(\%)=\frac{\frac{(A x+A y)}{2}-A(x+y)}{\frac{A x+A y}{2}} \times 100
$$

where $\mathrm{x}$ and $\mathrm{y}$ represent each of the two strains in the control 
tubes, and $(\mathrm{x}+\mathrm{y})$ the mixture.

\section{Results and Discussion}

\subsection{Antimicrobial Activity of Vaginal Labs}

In this study 25 rod and 15 coccii vaginal LABs were tested on antibacterial activity against $S$. aureus, E. coli, $C$. albicans and most active 4 cocci and 10 rod shaped LABs were isolated for farther investigation.

\subsection{Test on Biocompatibility}

The selected cocci were tested on biocompatibility with lactobacilli. Three of them were incompatible with all of lactobacilli (dates are not shown). The biocompatibility of the rest cocci: Lactococcus lactis GH 204 and lactobacilli are presented in Fig. 1.

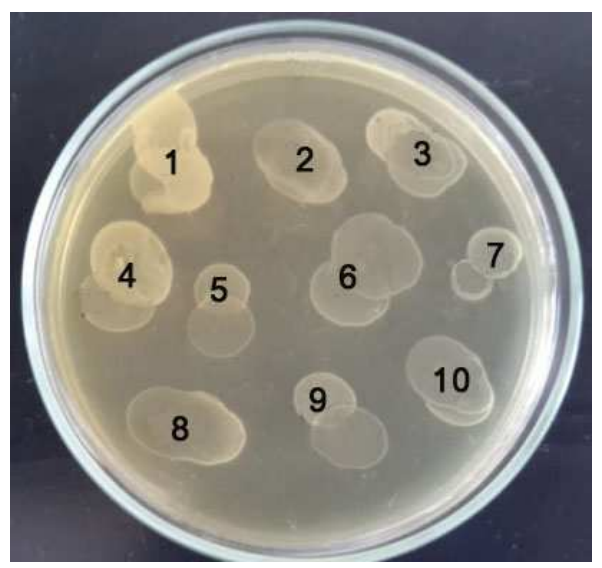

Fig 1. LABs biocompatibility assay.

The L. lactis GH 204 was biocompatible with two lactobacilli (numbers 2 and 8): Lactobacillus acidophilus GH 201 and Lactobacillus plantarum GH 202. For the further investigation was chosen the last one capable to produce high amount of $\mathrm{H} 2 \mathrm{O} 2$.

\subsection{Inhibition of Pathogens by Lactobacilli Supernatants}

Supernatants were obtained from separate and mixed overnight cultures L. lactis GH 204 and L. plantarum GH 202 grown in LAPTg broth at 370C. Supernatants antimicrobial activity was tested by disc diffusion method on test stains E. coli MDC 5003, C. albicans MDC 8013 and S. aureus MDC 5233 (Fig. 2 a, b, c).

The antibacterial activity of grown together overnight culture supernatant of L. lactis GH 204 and L. plantarum GH 202 is much powerful, than mono cultures (Fig. 2 a, b). Inhibit all test strains causing an increase in $15-25 \mathrm{~mm}$ zones. In the case of mixed culture antibacterial activity was highest: the $25-35 \mathrm{~mm}$. The antifungal activity of strain $L$. plantarum GH 202 and mixed culture supernatants are much higher, than antibacterial activity, probably due to $\mathrm{H}_{2} \mathrm{O}_{2}$ production (Fig. 2 c).

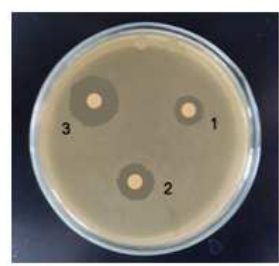

a)

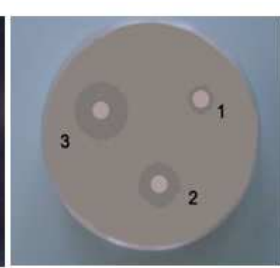

b)

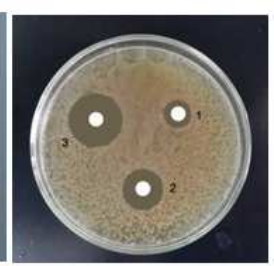

c)
Fig 2. Test strains: a) E. coli MDC 5003, b) S. aureus MDC 5233 and c) C. albicans MDC 8013, increasing inhibition by cultures supernatants $(1-L$. lactis GH 204, 2 - L. plantarum GH 202, 3 - mixed culture).

\subsection{The Growth Rate of the Cultures under Different PHs}

The cultures were grown in LAPTg at 370C with intensive agitation. OD of cultures were checked every $30 \mathrm{~min}$. The dates are presented in Fig. 3.
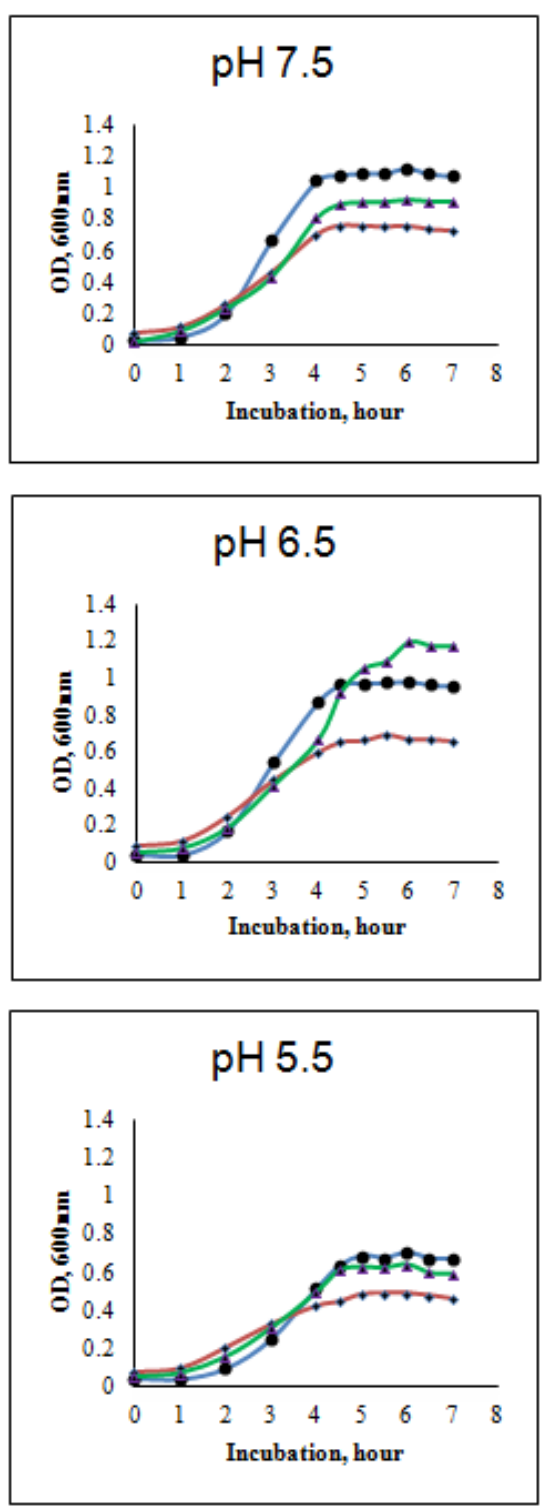

Fig 3. The growth rate of the cultures under different $p H s$ ( •-Lactococcus lactis GH 204, $\diamond$ - Lactobacillus plantarum GH 202, $\Delta$ - mix) 
As seen in the Fig. 3, L. lactis GH 204 growth rate at $\mathrm{pH}$ 7.5 excess the growth rate of L. plantarum GH 202 and leading in the mixed culture. At $\mathrm{pH} 6.5$ the synergism is obvious, because of the mixed culture growth more rapidly and accumulates a greater biomass, than the single cultures. In case of $\mathrm{pH} 5.5$ the growth rate of all cultures is low and there isn't significant difference between them.

\subsection{Sustainability of the Microbial Association}

The sustainability of the association was checked through serial subculturing in LAPTg broth with $\mathrm{pH}$ 7.6, which imitates changes between of the menstrual cycles and after sexual intercourses. The ratio of bacilli and cocci was estimated by plating on M16 agar over a period of 10 days and counting of small and large colonies.

Table 1. Ratio of the microbial association entities.

\begin{tabular}{llll}
\hline Subculturing* & $\mathbf{1}$ & $\mathbf{2}$ & $\mathbf{3 - 1 0}$ \\
\hline Rod/cocci & $1: 1$ & $3: 2$ & $2: 1$ \\
\hline
\end{tabular}

*The mean $\mathrm{pH}$ of overnight cultures was 4.5 .

As seen from Table 1, L. plantarum GH 202 and L. lactis GH 204 cultures ratio 2:1 become stable from the 3 rd day of 10 fold regular subculturing.

\subsection{Adhesion Properties of Vaginal Cultures}

Difficulties involved in studying bacterial adhesion in vivo, especially in humans, have led to the development of in vitro model systems for evaluation of strains adhesive potential $[1,5,10]$.

The MATS method was used to evaluate the hydrophobic/ hydrophilic cell surface properties of L. plantarum GH 202 and L. lactis GH 204. In this study, two solvents were tested for adherence to Lactobacteria: xylene (apolar solvent), chloroform (monopolar).

Table 2. Adhesion of L. plantarum GH 202 and L. lactis GH 204 to xylene and chloroform.

\begin{tabular}{lll}
\hline Culture & Chlorophorm & Xylene \\
\hline L. plantarum GH 202 & 29.47 & 33.68 \\
L. lactis GH 204 & 56.92 & 58.44 \\
\hline
\end{tabular}

The microbial adhesion to xylene and chloroform reflects cell surface hydrophobicity.

The results indicated that the both strains, particularly $L$. lactis GH 204 showed strong affinity for chloroform and xylene (Table 2). The adhesion property of L. lactis GH 204 is significantly higher in comparison to other strains of $L$. lactis isolated from human origin [14]

Adhesion, facilitated by bacterial cell surface hydrophobicity, is defined as the first phase of biofilm formation [8].

\subsection{Coaggregation of L. Plantarum GH 202 and L. Lactis GH 204}

Coaggregation of L. plantarum GH 202 with L. lactis GH 204 was examined. The high grade coaggregation of mixed cultures could increase their colonization potential. Results are expressed as the percentage reduction after $5 \mathrm{~h}$ in the absorbance of a mixed suspension compared with the individual suspension (Table 3 ).

Table 3. Coaggregation ability of L. plantarum GH 202 and L. lactis GH 204 after 5 incubation at room temperature in PBS (pH 7.2)

\begin{tabular}{lll}
\hline Culture & OD $_{\mathbf{6 0 0}}$ & Coaggregation \\
\hline L. plantarum & 0.172 & \\
L. lactis & 0.024 & $68,36 \%$ \\
L. plantarum + L. lactis & 0.031 & \\
\hline
\end{tabular}

A marked (68,36 \%) coaggregation was obtained between the strains. This property may be related to the formation of a mixed species biofilm since mixed species biofilms of $L$. monocytogenes and L. plantarum have been reported by Veen and Abee [16].

\section{Conclusion}

The association of L. plantarum GH 202 and L. lactis GH 204 strains has higher inhibitory activity than entities against bacteria associated with bacterial vaginosis and C. albicans.

The mixed culture growth rate and biomass accumulation are higher, than the mono cultures, which is very important for vaginal acidic $\mathrm{pH}$ rapid recovery violated by menses and sexual intercourse.

The association is stable during of vaginal $\mathrm{pH}$ changes caused by menses and sexual intercourses.

The coaggregation between L. plantarum GH 202 and $L$. lactis GH 204 strains could increase their colonization potential, when they used in mixed culture.

L. lactis GH 204 isolated from vagina in contrast to other lactococci has very high antimicrobial and adhesion properties. And it is the first time that lactococci were used as probiotic in vaginal colonization/recolonization practice.

\section{References}

[1] A. Ma“yra“-Ma“kinen, M. Manninen and H. Gyllenberg, The adherence of lactic acid bacteria to the columnar epithelial cells of pigs and calves. Journal of Applied Microbiology 55, 1983, 241-245.

[2] B. Larsen, G. R. Monif, Understanding the bacterial flora of the female genital tract. Clin Infect Dis, 32, 2001, 69-77.

[3] E. Wolters-Everhardt, J.M.J. Dony, W.H. Doesburg and J.H.M. De Pont, Buffering capacity of human semen. Fertil. Steril. 46, 1986, 114-119.

[4] G. Reid, J. Burton, Use of Lactobacillus to prevent infection by pathogenic bacteria. Microbes Infect, 4, 2002, 319-324.

[5] H. Kimoto, J. Kurisaki, T.M. Tsuji, S. Ohmomo and T. Okamoto, Lactococci as probiotic strains: adhesion to human enterocyte-like Caco-2 cells and tolerance to low $\mathrm{pH}$ and bile. Letters in Applied Microbiology 29, 1999, 313-316. 
88 Hrachya Garegin Hovhannisyan and Gohar Gagik Grigoryan: A New Sustainable Symbiotic Association of Lactic Acid Cocci and Bacilli for Colonization/Recolonization of Vagina and Prevention of Bacterial Vaginosis

[6] J.P. Lepargneur, V. Rousseau, Protective role of the Doderlein flora. J Gynecol Obstet Biol Reprod, 31, 2002, 485-494.

[7] M.N. Bellon-Fontaine, J. Rault and C.J. van Oss, Microbial adhesion to solvents: a novel method to determine the electrondonor/electron-acceptor or Lewis acid-base properties of microbialcells. Colloids and Surfaces 7, 1996, 47-53.

[8] O. Tresse, V. Lebret, T. Benezech, C. Faille, Comparative evaluation of adhesion, surface properties, and surface protein composition of Listeria monocytogenes strains after cultivation at constant $\mathrm{pH}$ of 5 and 7. Journal of Applied Microbiology, Oxford, v. 101, n. 1, 2006, 53-62.

[9] P. Marteau, J. C. Rambaud, Potential of using lactic acid bacteria for therapy and immunomodulation in man. FEMS Microbiol Rev., 12, 1993, 207-220.

[10] P.L. Conway and S. Kjellberg, Protein-mediated adhesion of Lactobacillus fermentum strain 737 to mouse stomach squamous epithelium. Journal of General Microbiology 135, 1989, 1175-1186.

[11] P.R. Murray, E.J. Baron, J.H. Jorgensen, M.A. Pfaller, R.H. Yolken, Manual of Clinical Microbiology, 8th Edition, ASM, Washington, D.C., 2003.

[12] P.S. Handley, D.W.S. Harty, J.E. Wyatt, C.R. Brown, J.P. Doran and A.C.C. Gibbs, A comparison of the adhesion, coaggregation and cell-surface hydrophobicity properties of fibrilar and fimbriate strains of Streptococcus salivarius. Journal of General Microbiology 133, 1987, 3207-3217.

[13] R.W. Jack, J.R. Tagg and B. Ray, Microbiol. Rev., 59, 2, 1995, 171-200.

[14] S.D. Todorov, M. Botes, S.T. Danova and L.M.T. Dicks, Probiotic properties of Lactococcus lactis ssp. lactis HV219, isolated from human vaginal secretions, Journal of Applied Microbiology 103, 2007, 629-639.

[15] S.S. Witkin, Bacterial flora of the female genital tract: function and immune regulation, Best Practice \& Research Clinical Obstetrics and Gynecology, Vol. 21, No. 3, 2007, 347-354.

[16] S.V. Veen, D.T. Abee, Mixed species biofilms of Listeria monocytogenes and Lactobacillus plantarum show enhanced resistance to benzalkonium chloride and peracetic acid. International Journal of Food Microbiology, Amsterdam, v. 144, n. $3,2011,421-431$.

[17] V. Redondo-Lopez, R.L. Cook, J.D. Sobel, Emerging role of lactobacilli in the control and maintenance of the vaginal bacterial microflora, Rev Infect Dis, 12, 1990, 856-872.

[18] V.L. Crow and P.K. Gopal, Cell surface differences of lactococcal strains. International Dairy Journal 5, 1995, 45-68.

[19] W.H. Masters and V. E. Johnson, In: Human sexual response, Bantam Books, Boston, Mass. 1966. 